\title{
Husserls Konzeption des Bewusstseins
}

\author{
CHRISTIAN BEYER
}

$\mathrm{Zu}$ den weithin anerkannten Leistungen Husserls gehört seine - nicht zuletzt in Göttingen entwickelte ${ }^{1}$ - Lehre vom ,inneren Zeitbewußtsein“. Sie bildet „ein Stück durchgeführter Phänomenologie“2 und beeindruckt durch ihre „oftmals geradezu mathematisch-formale[n] Beschreibungen“3. Gleichzeitig ist sie eine zentrale Komponente von Husserls allgemeiner Konzeption des je eigenen, aus der erstpersonalen Perspektive (,,ich“) zugänglichen Bewusstseins, dessen wesentliche Eigenschaften und Strukturen den Gegenstand seiner Phänomenologie bilden. Dieses Bewusstsein fließt gleichsam in einem Strom, dem Bewusstseinsleben in seiner Ganzheit. Für andere, nicht derart fließende Bewusstseinsformen interessiert sich Husserl nicht.

Einer der besten Kenner dieser Materie ist der dänische Husserl-Forscher Dan Zahavi. In einer Reihe von Aufsätzen und in seinem Buch Subjectivity and Selfhood unterzieht er Versuche, die auf Husserl zurückgehende Bewusstseinskonzeption im Sinne höherstufiger Repräsentationstheorien des Bewusstseins zu interpretieren, einer eindringlichen Kritik. ${ }^{4}$

Solche Theorien behaupten, dass bewusste mentale (d.h. psychische) $\mathrm{Zu}-$ stände oder Ereignisse - Husserl spricht von Erlebnissen - sich dadurch von unbewussten unterscheiden, dass sie ihrerseits intentionaler Gegenstand eines intentionalen Zustandes oder Ereignisses (einer „mentalen Repräsentation“) seitens des Erlebnissubjekts sind. Um was für höherstufige intentionale Zustände es sich dabei handelt - Perzeptionen, Urteilsakte oder bloß entsprechende Dispositionen - ist unter den Verfechtern höherstufiger Repräsentationstheorien kontrovers.

Versteht man diesen Vorschlag als These über die Natur - das „Wesen“ des Bewusstseins, so ist er sofort dem Einwand ausgesetzt, dass hier kein „,intrinsisches Merkmal“ (Zahavi), sondern lediglich eine „extrinsische“, relationale Eigenschaft erfasst wird, der sich Bewusstsein als solches unmöglich verdanken kann. ${ }^{5}$ Es muss vielmehr schon etwas Eigentümliches da sein, das intentionaler Gegenstand einer höherstufigen Repräsentation werden kann.

\footnotetext{
1 Vgl. Bernet 1985, S. XVII-LIII.

2 Künne 1986, S. 170.

3 Bernet 1982, S. XV.

4 Vgl. Zahavi 2002a; 2002b; 2004a; 2004b; 2005.

5 Vgl. Zavavi 2005, S. 20.
} 
Und dieses Eigentümliche besitzt als solches schon den in Frage stehenden Charakter des Bewusstseins:

Es ist eben ein Unding, von einem ,unbewußten' Inhalt zu sprechen, der erst nachträglich bewußt würde. Bewußtsein ist notwendig Bewußtsein in jeder seiner Phasen. ${ }^{6}$

Zahavi spricht sich daher für ein ,einstufiges“ Modell des Bewusstseins aus, welches er Husserl zuschreibt. ${ }^{7}$ Nach Husserl besitze Bewusstsein ,eine durchgängige Dimension der Selbstmanifestation“, die „,dem refle[kt]iven Selbstbewußtsein [vorausliegt] und es fundiert", und bei diesem ,,vor-refle[kt]iven Selbstbewußtsein" handle es sich um inneres Zeitbewusstsein. ${ }^{8}$

Ich halte diese These Zahavis über die intrinsische Natur des Bewusstseins nach Husserl für zutreffend; sie wird etwa durch das vorstehende Zitat belegt, wenn man seinen inhaltlichen Kontext (Analyse des inneren Zeitbewusstseins) berücksichtigt. ${ }^{\text {}}$ Was jedoch die strukturellen Eigenschaften des Bewusstseins (verstanden als Moment eines übergreifenden Erlebnisstroms) anlangt, so erscheint es mir aufschlussreich, Husserls Bewusstseinskonzeption nach Maßgabe einer höherstufigen Repräsentationstheorie zu interpretieren, die Zahavi nicht berücksichtigt, die aber zu vielem passt, was er über Bewusstsein und inneres Zeitbewusstsein à la Husserl sagt.

Ich denke dabei an eine dispositionale Spielart höherstufiger Repräsentationstheorien, der zufolge ein mentaler Zustand genau dann ein Erlebnis ist, wenn das Subjekt aufgrund dieses Zustandes zu dem (nicht-inferentiellen) ${ }^{10}$ Urteil disponiert wäre, dass es sich selbst gerade in diesem Zustand befindet, sofern es den Begriff eines solchen Zustandes (bereits) besäße. ${ }^{11}$ Der (vermutlich evolutionstheoretisch zu motivierende) ${ }^{12}$ Zusatz ,,sofern es den Begriff eines solchen Zustandes (bereits) besäße“ erlaubt es uns, im Einklang mit Husserl, ${ }^{13}$ auch kleinen Kindern und nicht-menschlichen Tieren (mit entsprechendem Lernpotential) Erlebnisse zuzuschreiben. Das Vorliegen einer höherstufige Urteilsdisposition des Inhalts, dass man sich gerade in dem-und-dem mentalen Zustand (z.B. visuelle Wahrnehmungserscheinung eines Baumes) befindet,

6 Husserl 1969, S. 119

7 Vgl. Zahavi 2005, S. 23.

8 Vgl. Zahavi 2002a, S. 712 f.

9 Für einen weiteren Textbeleg vgl. die unten zitierte Passage aus Husserl 1980, Beilage XII, S. $481 \mathrm{f}$.

10 Dieser Zusatz verhindert, dass wir von jemandem, der beispielsweise aufgrund der Kenntnis seiner äußeren Lebensumstände den Schluss zieht, dass er gerade traurig ist, sagen müssen, er erlebe Traurigkeit. Da das Metaurteil inferentiell zustande kommt, handelt es sich nicht um ein „reflektives“ oder introspektives (auf ,innerer Wahrnehmung" basierendes) Urteil.

11 Vgl. Beyer 2000, S. 112-119; 2006, Kap. 1.

12 Stichworte: Täuschungsverhalten, Fähigkeit zur Lösung komplexer Probleme.

13 Vgl. Husserl 1976, S. 73. 
aufgrund des so repräsentierten mentalen Zustands bildet lediglich den Normalfall, in dem der betreffende Zustand bewusst ist. Bei kleinen Kindern und höheren Tieren (mit einem Bewusstseinsleben) mögen derartige Urteilsdispositionen (noch) fehlen, da sie (noch) nicht über die nötigen (alltags-) psychologischen Begriffe verfügen. Sobald Bewusstsein im Erlebnisstrom eines vollbewussten Subjekts fließt, ist es jedoch in eine Struktur eingebettet, die es jederzeit für höherstufige Urteile des genannten Typs zugänglich macht.

Eine wichtige Komponente dieser Theorie besagt, dass Bewusstsein im relevanten Sinne stets in einem Gegenwartsbewusstsein gründet, und dabei könnte es sich um ,inneres Zeitbewußtsein“ im Sinne Husserls handeln. Wenn ich glaube, mich selbst gerade in dem-und-dem mentalen Zustand $\mathrm{zu}$ befinden, dann muss mir die Gegenwart in irgendeiner Weise präsent sein. Andernfalls wäre ich jetzt bestenfalls geistig abwesend (z.B. im Koma oder in einer traumlosen Schlafphase) und könnte schwerlich (intentional) etwas erleben. Husserl würde dieser Behauptung zustimmen. In Erfahrung und Urteil schreibt er:

Natürlich ist diese Zeit der Erlebnisse nicht die Zeit der in den Erlebnissen intentionalen Gegenständlichkeiten. Wenn z.B., während ich meine dingliche Umgebung wahrnehme, ein Erinnerungseinfall über mich kommt, und ich mich ihm gar zuwende, dann verschwindet nicht diese Wahrnehmungswelt [...] Die Erinnerung, in der ich nun lebe, bietet mir für das Erinnerte eine Zeit, die implizite orientiert ist zur Wahrnehmungsgegenwart. Aber das Erinnerte ist vergangen [...], während die Erinnerung als Erlebnis gleichzeitig ist mit dem Wahrnehmungserlebnis. ${ }^{14}$

Vielleicht übersieht Zahavi deshalb die Möglichkeit, Husserls Konzeption im Sinne der skizzierten dispositionalen höherstufigen Repräsentationstheorie aufzufassen, weil er die Aufzählung der drei relevanten Bewusstseinsbegriffe in $\$ 1$ der V. Logischen Untersuchung missversteht, von der er in seiner Interpretation (zurecht) ausgeht:

1. Bewußtsein als der gesamte reelle phänomenologische Bestand des empirischen Ich, als Verwebung der psychischen Erlebnisse in der Einheit des Erlebnisstroms.

2. Bewußtsein als inneres Gewahrwerden von eigenen psychichen Erlebnissen.

3. Bewußtsein als zusammenfassende Bezeichnung für jederlei $[\ldots]$, intentionale Erlebnisse ${ }^{6}{ }^{15}$

Husserl legt in den nachfolgenden Paragraphen dar, dass alles, was unter (2.) und (3.) fällt, auch der unter (1.) fallenden Gesamtheit zugehört, aber nicht umgekehrt; und dass wiederum alles, was dieser Gesamtheit zugehört, potentiell zu den ,psychischen Erlebnissen“ zählt, die in solchem Bewusstsein, das unter (2.) fällt, innerlich wahrgenommen werden. Bewusstsein im relevanten Sinne, sei es intentional oder nicht, ist demnach strukturell Teil des Erlebnisstroms und zugleich, in einem noch zu erläuternden Sinne, innerlich wahrnehmbar - der-

14 Husserl 1999, S. 205.

15 Husserl 1984, S. 356. Das Zitat entstammt der 2. Auflage der Logischen Untersuchungen. 
art dass es ,,innere Wahrnehmung“ und darauf basierende höherstufige Urteile motiviert, so dass normalerweise eine entsprechende Urteilsdisposition vorliegt.

Zahavi liest das Zitat anders. Nach seiner Interpretation wird unter (2.) die intrinsische, nicht-relationale Eigenschaft des Bewusstseins beschrieben, die sich näherhin als vor-reflektives Selbstbewußtsein bestimmen lässt. ${ }^{16}$ So kann die Rede vom ,inneren Gewahrwerden von eigenen psychischen Erlebnissen“ aber nicht gemeint sein, zumal Husserl den zweiten Bewusstseinsbegriff in $\int 5$ der V. Logischen Untersuchung ausdrücklich unter der Überschrift „Das ,innere" Bewußtsein als innere Wahrnehmung" thematisiert und in diesem Paragraphen dezidiert die „Annahme der kontinuierlichen Aktion innerer Wahrnehmung“ im Erlebnisstrom zurückweist. „Innere Wahrnehmung“ im hier infrage stehenden Sinne (zweiter Bewusstseinsbegriff) kann also nicht mit einem für das Bewusstsein als Moment des Erlebnisstroms wesentlichen und daher nie zu entbehrenden ,,vor-reflektiven Selbstbewußtsein“ zusammenfallen.

In den Logischen Untersuchungen und in den Ideen nimmt Husserl eine erste „Wesensanalyse“"17 des Bewusstseins vor, die mit einer dispositionalen höherstufigen Repräsentationstheorie in der Tat gut harmoniert. In den Ideen lesen wir:

Im cogito lebend, haben wir die cogitatio selbst nicht bewußt als intentionales Objekt; aber jederzeit kann sie dazu werden, zu ihrem Wesen gehört die prinzipielle Möglichkeit einer ,reflektiven‘ Blickwendung [...] Mit anderen Worten, jede kann zum Gegenstand einer sog. ,inneren Wahrnehmung' werden. ${ }^{18}$

Das typische Resultat einer solchen reflektiven Blickwendung dürfte ein introspektives - soll heißen: auf ,,innerer Wahrnehmung“ basierendes - Urteil des Inhalts sein, dass man sich selbst gerade in dem-und-dem mentalen Zustand befindet. Tiere sind nach Husserl allerdings außerstande, solche Urteile zu artikulieren - vielleicht sogar außerstande, sie zu fällen:

Ein ,waches‘ Ich können wir als ein solches definieren, das innerhalb seines Erlebnisstromes kontinuierlich Bewußtsein in der spezifischen Form des cogito vollzieht; was natürlich nicht meint, daß es diese Erlebnisse beständig, oder überhaupt, zu prädikativem Ausdruck bringt und zu bringen vermag. Es gibt ja auch tierische Ichsubjekte. ${ }^{19}$

Es gibt nach Husserls Auffassung auch nicht-intentionale Erlebnisse (etwa Schmerzempfindungen, aber auch Wahrnehmungsempfindungen alias hyletische Daten):

16 Vgl. Zahavi, 2002b, S. 52, 55-59. Der Hinweis auf das vor-reflektive Selbstbewusstsein findet sich auf S. 59.

17 Husserl 1976, S. 68.

18 Husserl 1976, S. 77; vgl. ebd., S. 87.

19 Husserl 1976, S. 73; meine Hervorhebung. 
Unter Erlebnissen im weitesten Sinne verstehen wir alles und jedes im Erlebnisstrom Vorfindliche; also nicht nur die intentionalen Erlebnisse, die aktuellen und potentiellen cogitationes, dieselben in ihrer vollen Konkretion genommen [...]. ${ }^{20}$

Ein intentionales Erlebnis gilt dabei als aktuelle (und nicht bloß potentielle) cogitatio, wenn es den gegenständlichen Vordergrund des Erlebnissubjekts (grob gesprochen: das, was sich im Fokus seiner Aufmerksamkeit befindet) fixiert.

Was den epistemischen Status der, ,inneren Wahrnehmung“ betrifft, die alle Erlebnisse im weitesten Sinne begleiten können muss, so betont Husserl, dass sie in ihrem jeweiligen intentionalen Gegenstand fundiert ist, also (als Erlebnis) nicht ohne das niederstufige Erlebnis existieren kann, auf das sie sich richtet. Darin unterscheidet sie sich von der (Wieder-)Erinnerung an ein (vermeintliches oder tatsächliches) Erlebnis. ${ }^{21}$ Von der äußeren Wahrnehmung, als deren „Repräsentant“ Husserl die Dingwahrnehmung behandelt, ${ }^{22}$ unterscheidet sie sich in der folgenden Hinsicht:

Das Ding nehmen wir dadurch wahr, daß es sich ,abschattet` nach allen gegebenenfalls ,wirklich" und eigentlich in die Wahrnehmung ,fallenden“ Bestimmtheiten. Ein Erlebnis schattet sich nicht ab. ${ }^{23}$

In gewissem Sinne ist die ,innere Wahrnehmung“ somit direkter auf ihren Gegenstand bezogen als die Dingwahrnehmung: es findet im Zuge dieser „Wahrnehmung“ keine objektivierende Auffassung von Empfindungsdaten (hyletischen Daten) statt. Dennoch liegen Husserl zufolge auch der ,inneren Wahrnehmung“ so etwas wie Empfindungsdaten (,Impressionen“) zugrunde:

Wenn es heißt, Impressionen, ebenso aber auch Reproduktionen [also anschauliche, empfindungsähnliche Momente von Vergegenwärtigungen wie z.B. Wiedererinnerungen; C.B.], klängen ab, so ist zu beachten, daß die Serie der Reproduktionen mit ihren Abklängen immerfort eine Einheit der Reproduktion ist und bleibt und daß es zum Wesen jeder Reproduktion gehört, daß sie auffaßbar ist als Darstellung von ... [...]. Das ist nun mißdeutlich. Aber wir müssen unterscheiden die Reproduktion selbst und die reproduktive Vorstellung, genau so, wie wir unterscheiden müssen die Impression selbst und die [...] Wahrnehmungsvorstellung. ${ }^{24}$

Husserl bezeichnet die allmählich abklingenden „Impressionen“, von denen hier die Rede ist, andernorts auch als „Urimpressionen“ bzw. „Retentionen“. ${ }^{25}$ Ich werde diese Begriffe gleich erläutern. Halten wir zunächst einmal fest, dass sie (1) das Empfindungsmaterial für die ,,innere Wahrnehmung“ eines

20 Husserl 1976, S. 74.

21 Vgl. Husserl 1976, S. 78f.

22 Vgl. Husserl 1976, S. 81.

23 Husserl 1976, S. 88.

24 Husserl 1969, S. $295 f$.

25 Vgl. Husserl 1980, S. 423. 
die Wahrnehmung fundierenden Erlebnisses abgeben sollen. Da sich das Erlebnis prinzipiell nicht abschattet (s.o.), müssen sie (2) dem „wahrgenommenen " Erlebnis selbst als Teil[e] untrennbar zugehören, statt - wie im Falle des Empfindungsmaterials der äußeren Wahrnehmung - nur in der Wahrnehmung, nicht aber in deren Gegenstand vorfindlich zu sein. Enger könnte der Kontakt zwischen Wahrnehmung und Wahrnehmungsobjekt nicht sein - und deswegen haben wir es hier mit einer ,absoluten Selbstgegebenheit" zu tun. (,Absolut“ - das steht bei Husserl oft im Gegensatz zu „subjektiv-relativ“ und besagt dann: nicht einseitig-perspektivisch; sondern dem intentionalen Gegenstand vollkommen adäquat. $)^{26}$ Ich komme auf diesen erkenntnistheoretischen Aspekt der ,inneren Wahrnehmung“ noch zurück.

Die ,innere Wahrnehmung“ muss nach Husserl alle meine Erlebnisse begleiten können, und im Normalfall dürfte dies bedeuten, dass ich zugleich automatisch zu einem Urteil des Inhalts disponiert bin, dass ich mich gerade in dem-und-dem mentalen Zustand befinde; einem Urteil, welches in der betreffenden ,inneren Wahrnehmung“ fundiert ist - und damit auch in dem sie fundierenden „wahrgenommenen“ Erlebnis mit all seinen Teilen. Wenn ich zu einem solchen introspektiven Urteil disponiert bin, dann gilt für mich ein kontrafaktisches Konditional:

Wenn die-und-die Umstände vorlägen (sc. „innere Wahrnehmung“ eines Erlebnisses der-und-der Sorte), dann würde ich normalerweise (sc. bei Besitz der erforderlichen Begriffe) ein introspektives Urteil des Inhalts fällen, dass ich mich gerade in einem mentalen Zustand der betreffenden Sorte befinde. ${ }^{27}$

Dieses Konditional gilt aufgrund einer (Wesens-)Gesetzmäßigkeit, die den Gehalt des introspektiven Urteils mit dem eigentümlichen Charakter des Erlebnisses verknüpft, in dem die zugrunde liegende ,innere Wahrnehmung“ und damit auch das introspektive Urteil fundiert ist. (Fundierung ist transitiv.) Dieser eigentümliche Charakter bestimmt nicht nur, dass es sich hierbei um ein Erlebnis der-und-der Sorte handelt, sondern auch, dass das Ichsubjekt dieses Erlebnis (als) soeben erlebt. Das Erlebnis besitzt für sein Subjekt einen präsentischen Charakter, der es für potentielle höherstufige Repräsentationen seitens dieses Subjekts zugänglich macht, die in einem besonders engen Kontakt zu ihrem Gegenstand stehen: eben „,innere Wahrnehmungen“ und introspektive Urteile. Dieser präsentische Charakter verdankt sich der „Urimpression “ innerhalb des „wahrgenommenen“ Erlebnisses, zu der wiederum - als eine Art „Kometenschweif“28 - „Retentionen“ gehören. Die „Urimpression“ sorgt dafür, dass das Erlebnis für das Ichsubjekt aus seiner Perspektive ,jetzt“ exis-

26 Vgl. etwa Husserl 1976, S. 92f.

27 Dabei ist zu beachten, dass die Wahrheit eines kontrafaktischen Konditionals (im hier relevanten Sinne) nicht die Falschheit seines Vordersatzes impliziert.

28 Husserl 1969, S. 295. 
tiert. ${ }^{29}$ Aus dem ,,jetzt“ wird blitzschnell ein ,soeben“: das Erlebnis wird in einer unmittelbaren Erinnerung des gerade Erlebten, einer Retention, aufbewahrt, an die sich wiederum eine Retention anschließt usw. Man denke z.B. daran, wie die schon gehörten Töne einer gerade erklingenden Melodie im Bewusstsein noch präsent sind, wenn sie auch allmählich ins Unbewusste absinken.

Dieses ,,innere Zeitbewußtsein“ macht das Erlebnis für die ,,innere Wahrnehmung“ und ggf. ein darin fundiertes introspektives Urteil, alias ,,reflektives Selbstbewußsein", zugänglich und bildet den zugrunde liegenden Motivationsfaktor. In diesem Sinne kann man es zurecht als ,,vor-reflektives Selbstbewußtsein" bezeichnen.

Unklar ist allerdings, ob und in welchem Sinne das ,,innere Zeitbewußtsein" nach Husserl eine nicht-setzende (also das Subjekt nicht auf die Existenz eines intentionalen Gegenstandes festlegende) Vorstufe einer Kenntnis des eigenen „Selbst“ enthält, wie Zahavi im Anschluss an Sartre behaupten möchte. ${ }^{30}$ Das Bewusstsein, welches sich im ,ich“-Sagen artikuliert, besitzt Husserl zufolge eine metarepräsentationale Struktur, ${ }^{31}$ es handelt sich dabei in seinen Augen um eine „Selbsterfahrung“ „,in schlichter Reflexion“.32 Husserl fügt hinzu, dass diese ,Selbsterfahrung [...] wie jede Erfahrung [...] bloss Hinmich-richten auf etwas [ist], das schon für mich da ist, schon bewusst ist und nur nicht thematisch erfahren ist, nicht Aufgemerktes". ${ }^{33}$ Daraus folgt jedoch nicht ohne weiteres, dass das laut Husserl schon vor-reflektiv bewusste „Selbst“ sich in irgendeiner nicht-setzenden Weise im ,,inneren Zeitbewußtsein“" manifestiert. Es gibt ja zahlreiche Formen des setzenden Bewusstseins, welche nicht den thematischen Fokus der Aufmerksamkeit fixieren, etwa Hintergrundwahrnehmungen im Falle der Beobachtung eines bestimmten Dinges.

Sofern die ,innere Wahrnehmung“ und das introspektive Urteil (das „,reflektive Selbstbewußtsein“, das „,cogito“) wirklich vollzogen werden, besitzen diese beiden Metarepräsentationen selber einen präsentischen Charakter,

29 Vgl. Husserl, 1980, S. 423: „Urimpression hat zum Inhalt das, was das Wort Jetzt besagt, wofern es im strengsten [sc. nicht-relationalen; C.B.] Sinne genommen wird. Jedes neues Jetzt ist Inhalt einer neuen Urimpression."

30 Vgl. Zahavi 2004a, S. 82ff.

31 Vgl. Husserl 1984, S. 813: „Wer, ich“ sagt, nennt sich nicht nur selbst, sondern er ist sich dieser Selbstnennung auch als solcher bewußt, und dieses Bewußtsein gehört wesentlich mit zum Bedeutungskonstituierenden des Wortes ,ich‘. [...] Der Hörende versteht es, sofern es ihm Anzeige für dieses ganze Bewußtseinsgebilde ist, also der Redende für ihn als jemand dasteht, der sich selbst, und zwar als, ich' nennt, d.i. sich als Gegenstand seiner als Selbsterfassung erkannten Selbsterfassung nennt." Diese Auffassung erscheint mir plausibel. Wenn jemand beispielsweise versichert: „Ich habe einen gebrochenen Fuß“, dann weiß er eo ipso, daß er sich mit ,ich“ auf sich selbst bezieht; er könnte sofort hinzufügen: „Ich spreche von mir selbst“ (vgl. Spitzley 2000, S. 40).

32 Hua XV, S. 492f.

33 Ebd. Vgl. dazu Zahavi 2002a, S. $705 f$. 
der sich mit der „Urimpression“ des sie fundierenden Erlebnisses deckt (,absolute Gleichzeitigkeit"), und sie können ihrerseits introspektiv repräsentiert werden. Dieser allen aktuell vollzogenen Elementen des Erlebnisstroms gemeinsame Aspekt der jeweiligen Gegenwart bezeichnet laut Husserl keine objektive zeitliche Bestimmung; und er ist natürlich irreduzibel subjektiv; er ist nur aus der egozentrischen Perspektive erfassbar.

Husserl thematisiert das so zugängliche Bewusstsein in einem grundlegenden erkenntnistheoretischen Zusammenhang, nämlich im Rahmen seines phänomenologischen Letztbegründungsprojekts: der sog. Konstitutionsanalyse. Er will - aus der erstpersonalen Perspektive - das „Problem der Transzendenz“ lösen:

[...] das Problem, wie das erkennende Bewußtsein in seinem Fluß mannigfach gestalteter und ineinander gewobener Erkenntnisakte sich selbst transzendieren und eine Gegenständlichkeit setzen und bestimmen kann, die in ihm nach keinem Bestandstïck reell $\mathrm{zu}$ finden ist, in ihm nie und nirgends $\mathrm{zu}$ absolut zweifelloser Selbstgegebenheit kommt, während sie doch dem Sinn der Naturerkenntnis gemäß an sich existieren soll, ob sie zufällig erkannt wird oder nicht. ${ }^{34}$

Es geht Husserl um eine „gegen allen vernünftigen Zweifel gesichert[e]“35 Begründung der Annahme objektiv (,,an sich“, „ob sie zufällig erkannt [werden] oder nicht") existierender, und damit die "Selbstgegebenheit" eigenen Bewusstseins transzendierender, raumzeitlicher Gegenstände. Sämtliche Voraussetzungen, die auf dieser Annahme beruhen, werden eingeklammert. Erlaubt sind zunächst nur Konstatierungen ,absolut zweifellose[r] Selbstgegebenheiten" des eigenen Bewusstseins. (Man könnte diese Herangehensweise als methodischen Fundamentalismus bezeichnen.) „Absolut zweifellos“ - das besagt: selbst unter der methodischen Vorgabe größtmöglicher Vorurteilslosigkeit und Anschaulichkeit keiner weiteren Begründung bedürftig und fähig. Diesen Test bestehen laut Husserl die Gegebenheiten des inneren Zeitbewusstseins, die jegliches Bewusstsein (im relevanten Sinne) fundierenden „Zeitempfindungen", soweit die adäquate Beschreibung dieser Daten von der in Frage stehenden Annahme objektiv existierender Gegenstände unabhängig ist. Aufgrund dieser Gegebenheiten konstituieren sich immanente Erlebnischaraktere, denen sich die oben erwähnte ,,absolute Selbstgegebenheit“ der darin fundierten ,,inneren Wahrnehmung" verdankt. Erst in der phänomenologischen Konstitutionsanalyse treten diese Gegebenheiten explizit hervor.

Ich erwähnte bereits, dass diese Konstitutionsanalyse mit einem methodischen Fundamentalismus beginnt: alle Objektivitätsannahmen werden eingeklammert, nur „absolute Selbstgegebenheiten“ des eigenen Bewusstseins gelten als unproblematisch. Husserl nennt diese Gegebenheiten auch „Phänomene im absoluten Sinne“ und definiert sie als „Erlebnisse vor der Objektivation“. ${ }^{36}$

34 Husserl 1969, S. 345.

35 Husserl 1969, S. 344.

36 Husserl 1969, S. 295. 
Was bleibt übrig, wenn nur potentielle Gegenstände innerer Wahrnehmung unter Absehung von jedweder objektivierender Auffassung, welche den (perspektivischen) Bezug auf Transzendentes ermöglichen würde, die hohen epistemischen Standards des methodischen Fundamentalismus erfüllen? Husserls Antwort lautet: Urimpressionen, die er auch „Zeitstellenrepräsentanten“ nennt, ${ }^{37}$ und damit verbundene (das absolute Jetzt „extendierende“) Retentionen; also das dem Erlebnis einwohnende Bewusstsein der Gegenwart und der sich daran unmittelbar anschließenden Vergangenheit, aber in Abstraktion von jeder darin ggf. fundierten objektiven Zeitauffassung:

Dieses absolute Werden ist das Fundament für alle Zeitauffassung, nicht aber sie selbst. [...] Objektivität ist noch nicht gegeben in bloßen ,Inhalten' und bloßem Fluß der Inhalte. Wo das Bewußtsein nicht Synthesis vollzieht und z.B. in der Dauer nicht Identität eines Dauernden identifizierend setzt, da ist auch keine Dauer, sondern nur ein bestimmt zu charakterisierender Fluß von Inhalten. [...] Ohne Identifizierung und Unterscheidung, ohne Jetzt-Setzung, Vergangenheits-Setzung, Zukunfts-Setzung etc. bleibt der absolute ,Inhalt' blind, bedeutet nicht objektives Sein, nicht Dauern etc. ${ }^{38}$

Die ,absolute Selbstgegebenheit“ dieser Inhalte reicht weiter als das urimpressionale ,Jetzt[, das im stetigen Fluß ist]“", es umfasst auch das retentionale „Eben-gewesen-sein““. ${ }^{39}$ Als Beispiel führt Husserl die Wahrnehmungserscheinung eines dauernden Tons an. Auch die darin involvierten Retentionen sind ,gegen jeden vernünftigen Zweifel gesichert". ${ }^{40}$

Wie ist es unter den Bedingungen des methodischen Fundamentalismus um die „Wiedererinnerung“ bestellt? Husserl argumentiert, dass sie „nicht immer vernünftig zweifelhaft ist":

[...] nämlich wenn wir durch einen kontinuierlichen Weg der Erinnerung von ihr [der Wiedererinnerung; C.B.] zum Jetzt und vom Jetzt durch Retention hindurch und durch stetig wiederauflebende Retention hindurch wieder zurückgelangen zu dem in der Wiedererinnerung Gesetzen. ${ }^{41}$

Auf diese Weise kann ich mir in radikal-phänomenologischer Einstellung klarmachen, dass eine Bewusstseinskette dezent zu dem durch Wiedererinnerung abgerufenen Erlebnis zurückführt. Nicht nur die Gegenwart, sondern auch die Vergangenheit des eigenen Bewusstseinsflusses ist so gegen vernünftigen Zweifel gesichert.

Die weiteren Schritte der Konstitutionsanalyse, in denen die Erlebnisart der Einfühlung in fremdes Bewusstseinsleben eine entscheidende Rolle spielt, ${ }^{42}$

37 Husserl 1980, S. 422.

38 Husserl 1969, S. $296 f$.

39 Husserl 1969, S. 343f.

40 Husserl 1969, S. 344.

41 Husserl 1969, S. 345.

42 Vgl. Hua VII, S. 435. 
dienen u.a. dazu, auch die objektivierenden Auffassungen, von denen im vorletzten Zitat die Rede ist, gegen vernünftigen Zweifel abzusichern. ${ }^{43}$ Wie das im Einzelnen geschieht, und wie weit der methodische Fundamentalismus dabei reicht, ist für meine Zwecke ohne Belang. Es geht mir ja um diejenigen Bewusstseinsdaten, die Husserl zufolge in der fundamentalistischen Phase der Konstitutionsanalyse ins Blickfeld geraten.

Diese Daten besitzen einen präsentischen und, wie wir jetzt sagen können, auch auf die abgeflossene Gegenwart bezüglichen Charakter. Jedes Erlebnis enthält solche Daten und ist in ihnen fundiert. Diese Daten sind dafür verantwortlich, dass das Erlebnis fließt, also Teil eines Bewusstseinsflusses ist. ${ }^{44}$ Dieser Fluss ist klar strukturiert, er besteht aus Phasen, die zueinander in Relationen des „Vorher und Nachher“ und des Zugleich stehen. ${ }^{45}$ Es handelt sich bei diesen Phasen um Empfindungen, die fundierender Bestandteil des betreffenden Erlebnisses sowohl als auch der ,,inneren Wahrnehmung“ des Erlebnisses sind. Dank der Relationen des Vorher/Zugleich/Nachher, in denen diese Empfindungen $\mathrm{zu}$ den anderen Flussphasen stehen, generieren sie die Möglichkeit, die in ihnen fundierten Erlebnisse eindeutig zu identifizieren:

Alle Erlebnisse eines Ich [...] sind konstituiert im absoluten Fluß des inneren Zeitbewußtseins und haben in ihm ihre absolute Lage und Einmaligkeit, ihr einmaliges Auftreten im absoluten Jetzt, worauf sie retentional abklingen und in die Vergangenheit zurücksinken. ${ }^{46}$

In jeder Phase des (überall gleich strukturierten) Flusses kann sich daher erneut ein Erlebnis durch „,innere Wahrnehmung" als eine „,immanente Einheit“ (Husserl) konstituieren, die dank ihrer ,absolute[n] Lage und Einmaligkeit“" und (wie man wegen der Möglichkeit absolut gleichzeitiger Erlebnisse hinzufügen sollte) ihrer Aktqualität von allen übrigen Einheiten des Flusses verschieden ist. In diesem Sinne begleitet die ,innere Wahrnehmung“ potentiell jedes soeben dahinfließende Erlebnis. Husserl bezeichnet die zugrunde liegenden absoluten Zeitempfindungen (in terminologischem Anschluss an Brentano) auch als ,inneres Bewußtsein“ oder „Wahrnehmen“ des betreffenden Erlebnisses, jetzt aber in einem nicht-intentionalen Sinne des Wortes:

Jedes Erlebnis ist ,empfunden' [...] (inneres Bewußtsein), wenn auch natürlich nicht gesetzt, gemeint. [...] Freilich scheint das auf einen unendlichen Regreß zurückzufuihren. [...] Dagegen ist zu sagen: [...] Jedes Erlebnis, das der Blick treffen kann, gibt sich als ein dauerndes, dahinfließendes, sich so und so veränderndes. Und das macht nicht der meinende Blick, er blickt nur darauf hin.

43 Vgl. Husserl 1999, S. 192: „In [der Einfühlung] konstituiert sich eine intersubjektiv gemeinsame objektive Zeit, in die alles Individuelle an Erlebnissen und an intentionalen Gegenständlichkeiten muß eingeordnet werden können."

44 Vgl. Husserl 1980, S. 430f.

45 Vgl. Husserl 1980, S. 430.

46 Husserl 1999, S. 205. 
Dieses gegenwärtige, jetzige, dauernde Erlebnis ist schon, wie wir durch Blickänderung finden können, eine ,Einheit des inneren Bewußtseins', des Zeitbewußtseins [...] mit seinen Phasen der fließenden Retentionen und Protentionen. ${ }^{47}$

Das ,innere Bewußtsein“, welches jedem Erlebnis als fundierende Komponente einwohnt, disponiert eben nur zu ,inneren Wahrnehmungen“ des Erlebnisses (im intentionalen Sinne des Wortes „Wahrnehmung“) und zu darin fundierten introspektiven Urteilen. Es sorgt dafür, dass ,,der Blick“ das im absoluten Fluss bereits existierende Erlebnis als gerade dahinfließend „treffen kann“. In diesem Sinne gehört zu jedem Erlebnis eine dispositionale Metarepräsentation. Auf diese Weise entgeht Husserl dem Standardeinwand gegen ,aktualistische“ Varianten höherstufiger Repräsentationstheorien, wonach die darin postulierten Metaperzeptionen oder Metaurteile einen unendlichen Regress zunehmend höherstufiger Repräsentationen in Gang setzen würden.

Zahavi spricht im Zusammenhang mit dem, was Husserl im vorstehenden Zitat ,inneres Bewußtsein“ nennt, von passiver, impliziter Selbstaffektion und zitiert ein spätes Manuskript (C 16), in dem Husserl feststellt, er werde ,unaufhörlich durch [sich] selbst affiziert" ${ }^{48}$ Eine Möglichkeit, diesen erläuterungsbedürftigen Gedanken auszubuchstabieren, bietet die skizzierte dispositionale Spielart der höherstufigen Repräsentationstheorie. ${ }^{49}$ Die fraglichen Dispositionen werden in introspektiven Urteilen aktualisiert, ihr Gehalt weist also einen erstpersonalen Aspekt auf. Wenn Husserls Analysen zutreffen, dann gibt es etwas, das diese Dispositionen fundiert und erklärlich macht: das dem Bewusstsein immanente Zeitempfinden. Dieses ,innere Bewußtsein“ evoziert nicht ständig ,,innere Wahrnehmungen“ und in weiterer Folge introspektive Urteile - es steht aber durchgängig bereit, um solche reflektiven Akte zu motivieren und gleichzeitig zu fundieren. Ohne entsprechende höherstufige Urteilsdispositionen bliebe das ,innere Bewußtsein“ blind, und es wäre kaum angemessen, es als „vor-reflektives Selbstbewußtsein“ anzusprechen.

47 Husserl 1980, Beilage XII, S. 481f. Protentionen hatte ich bislang nicht erwähnt; und werde es auch im Folgenden nicht tun.

48 Vgl. Zahavi 2002a, S. 720f.

49 Umgekehrt ist Husserls Konzeption des „,inneren Bewußtseins“ geeignet, empirische Hypothesen der dispositionalen höherstufigen Repräsentationstheorie phänomenologisch zu motivieren. So postuliert Peter Carruthers' Version dieser Theorie neben einer Funktionseinheit namens „N-box“, welche im Wahrnehmungsfalle Empfindungsdaten (,Perzepte“) direkt in Körperbewegungen umsetzt, eine „C-Box“ (,C“ steht für consciousness), die solche Daten automatisch für (introspektive) Metaurteile zugänglich macht, die dann ihrerseits körperliches Verhalten (mit-)verursachen können (vgl. Carruthers 2000, S. 228, Abb. 8.1). Warum Empfindungsdaten regelmäßig in die CBox eingefüttert werden und damit jederzeit zu introspektiven Urteilen führen können, erklärt diese Version der Theorie freilich nicht. Hier greift Husserls These von der ständig wiederkehrenden Struktur „Urimpression-Retention“. 


\section{Literaturverzeichnis}

Bernet, Rudolf (1982), „Einleitung“, in: Edmund Husserl, Texte zur Phänomenologie des inneren Zeitbewußtseins (1893-1917), Hamburg: Meiner, S. XI-LXVII.

Beyer, Christian (2000), Intentionalität und Referenz, Paderborn: mentis.

Beyer, Christian (2006), Subjektivität, Intersubjektivität, Personalität, Berlin/New York: De Gruyter.

Carruthers, Peter (2000), Phenomenal Consciousness, Cambridge: Cambridge University Press.

Husserl, Edmund (1969), Zur Phänomenologie des inneren Zeitbewusstseins (1893-1917), hrsg. von Rudolf Boehm. Nachdruck der 2. verb. Auflage, Den Haag: Nijhoff.

Husserl, Edmund (1973), Zur Phänomenologie der Intersubjektivität. Texte aus dem Nachlass. Dritter Teil: 1929-1935, hrsg. von Iso Kern, Den Haag: Nijhoff.

Husserl, Edmund (1976), Ideen zu einer reinen Phänomenologie und phänomenologischen Philosophie. Erstes Buch: Allgemeine Einführung in die reine Phänomenologie. 1. Halbband, hrsg. von Karl Schuhmann, Den Haag: Nijhoff.

Husserl, Edmund (1980), Vorlesungen zur Phänomenologie des inneren Zeitbewußtseins, 2. Aufl. Tübingen: Niemeyer.

Husserl, Edmund (1984), Logische Untersuchungen. Zweiter Band: Untersuchungen zur Phänomenologie und Theorie der Erkenntnis, hrsg. von Ursula Panzer, Den Haag: Nijhoff.

Husserl, Edmund (1999), Erfahrung und Urteil, 7. Aufl. Hamburg: Meiner.

Künne, Wolfgang (1986), „Edmund Husserl: Intentionalität“, in: Josef Speck (Hg.), Grundprobleme der großen Philosophen: Philosophie der Neuzeit IV, Göttingen: Vandenhoeck \& Ruprecht, S. 165-215.

Spitzley, Thomas (2000), Facetten des ,ich”, Paderborn: mentis.

Zahavi, Dan (2005), Subjectivity and Selfhood, Cambridge/Mass.: MIT Press.

Zahavi, Dan (2002a), „Husserl und das Problem des vor-reflexiven Selbstbewußtseins“, in: Heinrich Hüni und Peter Trawny (Hg.), Die erscheinende Welt, Berlin: Duncker \& Humblot, S, 697-724.

Zahavi, Dan (2002b), „,The Three Concepts of Consciousness in Logische Untersuchungen“, in: Husserl Studies 18, S. 51-64.

Zahavi, Dan (2004a), „Back to Brentano?“, in: Journal of Consciousness Studies 11, Nr. 10-11, S. 66-87.

Zahavi, Dan (2004b), „Time and Consciousness in the Bernau Manuscripts“, in: Husserl Studies 20, S. 99-118. 